\title{
Annotation tool hits a milestone
}

\section{Users of the open-source Hypotheis software have posted more than 5 million comments on the scholarly web.}

\section{BY RINA SHAIKH-LESKO}

$\mathrm{T}$ he team behind Hypothesis, an opensource software tool that allows people to annotate web pages, announced in March that its users had collectively posted more than 5 million comments across the scholarly web since the tool was launched in 2011. That's up from about 220,000 total comments in 2015 (see 'Comment counts'). The company has grown from 26,000 registered users to 215,000 over the same period.

"Five million annotations is a major milestone for us, and something we've worked hard to achieve," says Hypothesis founder, Dan Whaley, who is based in San Francisco, California.

Hypothesis is one of a handful of tools others include A.nnotate, Diigo, PaperHive and Remarq - that allow users to mark up and comment on any web page or online PDF, and to see annotations made by others. Hypothesis focuses on the scientific literature, and has, over the past few years, partnered with publishers, societies and educational institutes, including the journal eLife, the American Geophysical Union, the American Society of Plant Biologists and Cambridge University Press, to integrate that functionality into article pages and editorial processes. Working with eLife, for example, the service built tools to allow staff to moderate comments, and to track and authenticate users through ORCID - a digital identifier for academic authors. The journal's

\section{COMMENT COUNTS}

Users of the software tool Hypothesis have collectively posted more than 5 million annotations since 2015.

6

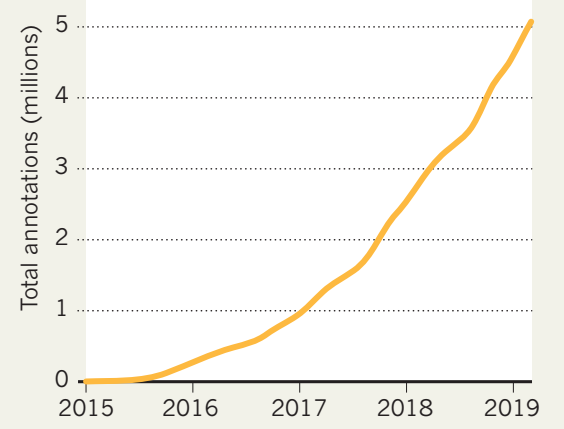

readers have used the annotation tool to note minor errors and to flag up other papers that agree or disagree with conclusions. And editorial staff have used it to contribute to the dialogue. In the 15 months since eLife introduced the system, more than 6,000 annotations have been posted on nearly 2,000 documents, although about half of those were made by eLife's automated curation assistant, SciBot.

"I don't think the quality of the dialogue in [the previous commenting system] was ever as constructive or as good as it is within the Hypothesis ecosys-

\section{"Five million}

annotations is a major milestone forus, and something we've worked hard to tem," says Giuliano Maciocci, head of product and user experience at eLife in Cambridge, UK. That, he says, is probably because annotations are positioned on top or next to the text to which they refer, instead of at the bottom of the page as they are in conventional commenting systems.

Some publishers have integrated annotations into their peer-review processes. In the American Geophysical Union's system, for instance, comments can be organized by reviewer or editor, and by importance. Reviewers can see only their own comments, whereas editors can see all comments from reviewers and manuscript authors.

And some publishers are taking advantage of the tool's enhanced group feature, which allows users to create groups that are open (available to all users), private (anyone can read, but only invited members can annotate) or restricted (only invited members can see or add annotations). Last December, the American Society of Plant Biologists launched an open group for annotations on its journal Plant Cell; it has logged almost 30 comments.

Hypothesis is accessed using a Chrome extension, a bookmarklet, or the via.hypothes.is proxy server. In February 2017, the World Wide Web Consortium, or W3C, the international standards body for the web, formally recommended a web-annotation standard that allows developers to make annotation a native feature of their browsers. So far, none has done so.

Rina Shaikh-Lesko is a science writer based in San Jose, California.
Are

you a

scientist

mentor or

mentee?

Read our cross-

sector mentoring

series for advice

and support

go.nature.com/

mentoringresource 